\title{
Image Steganalysis Based on Wavelet Packet Decomposition
}

\author{
Lei Yu \\ Electronics Department of Engineering University of the Armed Police Force, Xi' an 710086, China \\ ly1a2b3c@163.com
}

Keywords: steganalysis; wavelet packet decomposition

\begin{abstract}
In the paper, it proposed an image steganalysis method using wavelet packet decomposition. First, it decomposed the image using wavelet packet decomposition. Second, it extracted the higher-order statistics of its wavelet packet subbands as features. The experiments on F5 and MB steganography showed the method can detect stego images reliably.
\end{abstract}

\section{Introduction}

Steganography is the art and science of "invisible" communication, which is to conceal the very existence of hidden messages. Steganalysis is the art of detecting the presence of hidden messages, which is the counter problem to steganography. It has special steganalysis and universal steganalysis. The former is mainly for a specific steganographic method. It has good detection results, but bad flexibility and scalability. The latter is not for a specific steganographic method. It constructs a universal detector, detects various steganographic images, has good generality, but has bad detection for a specific steganographic method. This paper focuses on universal steganalysis.

Various steganalysis methods have been proposed for attacking steganographic algorithms. In universal steganalysis methods, Farid et al. proposed a universal steganalyzer based on image's higher-order statistics ${ }^{[1][2]}$. First, it decomposed the image using wavelet packet decomposition. Second, it extracted the higher-order statistics of its wavelet packet subbands. Steganography is equivalent to adding noise signals to a cover image. These noise signals usually distribute over high frequency coefficient of wavelet subbands. But the method of Farid did not make further decomposition to frequency subbands in feature extraction. The excavation of noisy signals was not enough.

In this paper, a new universal steganalysis method was proposed to effectively detect steganographic images using wavelet packet decomposition. First, it decomposed the image using wavelet packet decomposition. Second, it extracted the higher-order statistics of its wavelet packet subbands as features. The experiments on F5 and MB steganography showed the method can detect stego images reliably.

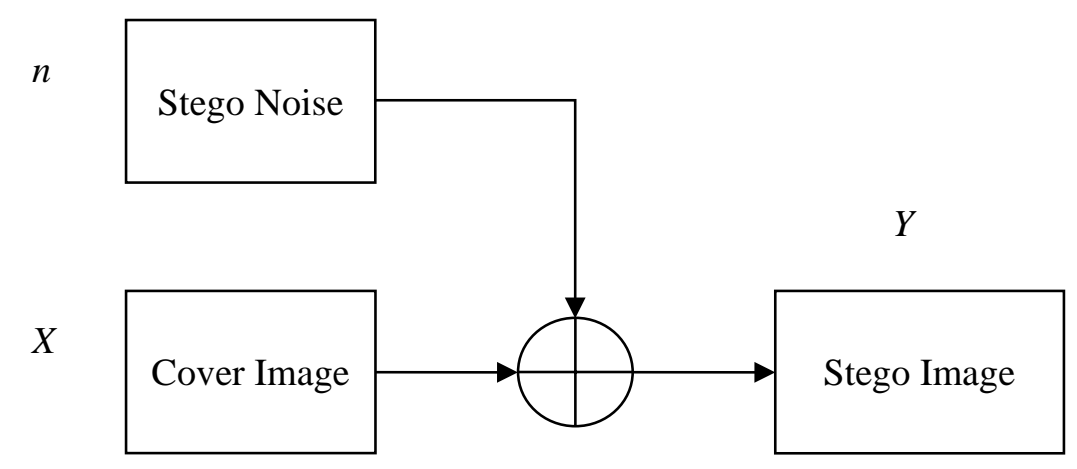

Figure 1 Additive nosie model 


\section{Feature Generation}

\subsection{Additive noise model}

Harmsen et al. proposed additive nosie model as shown in Figure $1^{[3]}$. In this model, the process of information hiding is equivalent to get stego image in the cover image with stego noise. Assume that the pixel values at the location $(i, j)$ of the cover image are represented by random variables $X_{i j}$, the location $(i, j)$ of the stego image are represented by random variables $Y_{i j}$, the location $(i, j)$ of the stego nosie are represented by random variables $n_{i j}$. So the steganography process can be represented as:

$$
Y_{\mathrm{ij}}=X_{i j}+n_{i j}
$$

$0 \leq i \leq M-1,0 \leq j \leq N-1, M \times N$ is the size of the image. This model is applicable to most existing steganography methods. Inspired by this model, in order to locate the influence of the cover signal in the stego image, obtain a signal that is more sensitive to embedded changes, we estimate the stego noise firstly from the stego image. In the detection process, the cover image is not known, so the stego noise can not be obtained by subtracting the cover image with the stego image. Usually, the high frequency subband of the wavelet decomposition of the stego image can be as an estimate of the stego image. Therefore, it can be seen that the high frequency subbands of wavelet coefficients play more important role in steganalysis than the low frequency subbands. The method of Farid only decomposed the image using wavelet decomposition, this is not enough to excavate the noise signal in the image. This paper will decompose the image using wavelet packet decomposition, in order to overcome the disadvantage of wavelet decomposition to high frequency subbands.

\subsection{Wavelet packet decomposition}

Wavelet decomposition decomposes the signal into low frequency and high frequency parts. The first layer decomposition decomposes the signal into low frequency and high frequency parts. The next layer decomposition decomposes the low frequency parts into low frequency and high frequency parts and so on. But wavelet packet decomposition is not. It doesn't just decompose the low frequency parts, but also the high frequency parts. So, it overcomes the disadvantage of poor resolution of high frequency parts and can decompose the signal in the full band range. It has a stronger advantage in the characterization of the signal.

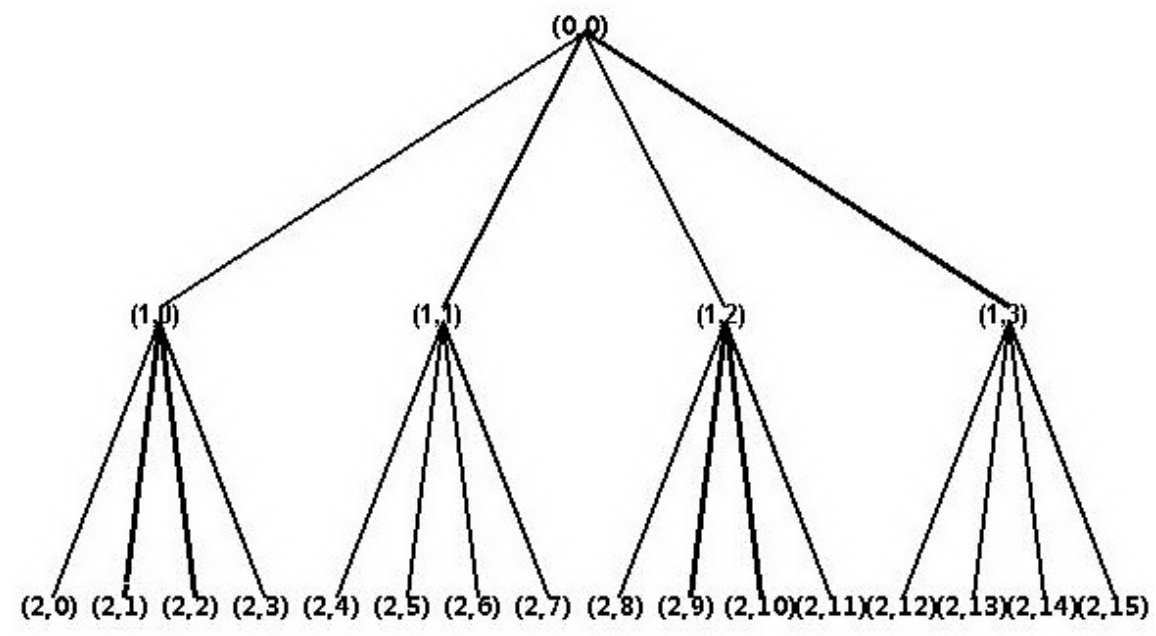

Figure 2 Tree structure of two-layer wavelet packet decomposition

In the paper, two-layer wavelet packet decomposition is used as shown in Figure 2. Because the information entropy of the third layer is too low. So the effect on manuscripts steganalysis is negligible. In most blind steganalysis methods, the higher-order statistics of an image and its other representations are used as features for classification ${ }^{[1][2][4][5]}$.

The statistical moments of the characteristic function of the histogram of the image and their 
wavelet subbands were proved to be very effective features and were applied in a series of previous work $^{[4][5]}$. Here, this paper drew on this method, and utilized the statistical moments of the characteristic function (CF) of the histogram of the image and their wavelet packet subbands as features, defined as

$$
\mathbf{M}_{n}=\sum_{k=0}^{L-1} \sin ^{n}\left(\frac{\pi k}{L}\right)|H(k)|, n=1,2,3
$$

Where $n$ is the order of moments, $L$ is the length of the characteristic function of the histogram (i.e. the discrete Fourier transform of the histogram) of the image and their wavelet packet subbands, $k$ is the $k$-th frequency in discrete Fourier transform (DFT), $|H(k)|$ is the amplitude of DFT of the histogram of the image and their wavelet packet subbands $h(m)$.

The discrete Fourier transform of the histogram is defined as follows,

$$
\begin{aligned}
& H(k)=\sum_{m=0}^{M-1} h(m) \exp \left\{\frac{j 2 \pi m k}{K}\right\} \\
& 0 \leq m \leq M-1
\end{aligned}
$$

Where, $K=2^{\left\lceil\log _{2} M\right\rceil}, h(m)$ represents the histogram of the image and their wavelet packet subbands, $M$ represents the longth of the histogram. The first three order moments of the characteristic function of the histogram of the image and their wavelet packet subbands were extracted as features for steganalysis. There were twenty-one subbands in total, so 63-D feature vector was got.

Fisher linear discriminator(FLD) was taken as the classifier in the experiments. This classifier is a classical classifier for solving binary classification. It has the advantages of less parameter setting and fast calculation. Its idea is to project the d dimensional feature space onto a straight line and form one-dimensional space. Normally, if the sample is linear separable, it can always find a direction in which the projection of the sample can be best separated from the straight line in this direction. Before applying the classifier, an important step is to scale the features to [-1,1]. The purpose of this scaling step is to prevent features with large numerical ranges from dominating those with small numerical ranges, avoid numerical ill-conditioning, and dramatically improve classification accuracy.

\section{Simulation experiment and result analysis}

$\mathrm{F}^{[6]}$ and $\mathrm{MB}^{[7]}$ were tested in the simulation experiment. The experiment used UCID image library ${ }^{[8]}$. This image library consists of 1338 images which are 24-bit color TIFF images without JPEG compression. Image size is $512 * 384$ or $384 * 512$. The content of the image library involves a wide range of nature, scenery, figures, architecture, etc. 1000 images were randomly selected from the image library. These images were converted to grayscale JPEG images with a quality factor of 80 by Matlab. Steganography was done with $10 \%$ and $50 \%$ of the full capacity in F5 and MB and the corresponding stego images were generated for testing. Choosed 500 images for FLD classifier training and the rest for testing.

As can be seen from table 1 , the detection rate of this method was about $1 \%$ to $2 \%$ higher than that of the Farid's. Analyze the causes, Farid's method decomposed the image with wavelet decomposition. Wavelet decomposition only decomposes the low frequency part, and does not decompose the high frequency part containing more noisy signals. But in this paper, wavelet packet was used to decompose the image. It can overcome the disadvantage of poor resolution of high frequency parts and decompose the signal in the full band range. It has a stronger advantage in the characterization of the signal and improves the validity of the features. 
Table 1 Comparison of two methods for detection results (\%)

\begin{tabular}{|c|c|c|c|}
\hline Steganalysis method & $\begin{array}{l}\text { Embedded } \\
\text { capacity }\end{array}$ & Farid's & This paper \\
\hline \multirow{2}{*}{ F5 } & $10 \%$ & 74.1 & 76.1 \\
\hline & $50 \%$ & 81.5 & 82.2 \\
\hline \multirow{2}{*}{ MB } & $10 \%$ & 65.8 & 67.5 \\
\hline & $50 \%$ & 74.2 & 75.1 \\
\hline
\end{tabular}

\section{Summary}

In this paper, a new universal steganalysis method was proposed using wavelet packet decomposition. This method decomposed the image using wavelet packet decomposition which could decompose the signal in the full band range. And it extracted the higher-order statistics of its wavelet packet subbands as features. FLD was taken as the classifier. The experiments on F5 and MB steganography showed the method can detect stego images reliably. But it can be seen, the overall detection rate of this method is not particularly high, both of which are not more than $90 \%$, and there is still a lot of room for improvement.

\section{References}

[1] Farid H. Detecting hidden messages using higher-order statistical models. The 5th International Conference on Image Processing. New York: Springer, 2002:905-908.

[2] Farid H, Siwei L. Detecting hidden message using higher-order statistics and support vector machine. The 5th Information Hiding Workshop. New York: Springer, 2002: 131-142.

[3] Harmsen J, Pearlman W. Steganalysis of additive-noise modelable information hiding. Security Watermarking Multimedia Contents. New York: SPIE, 2003: 131-142.

[4] Xuan G R, Shi Y Q, Gao J J, et al. Steganalysis based on multiple features formed by statistical moments of wavelet characteristic functions. The 7th Information Hiding Workshop. Barcelona: Springer, 2005:262-277.

[5] Wang Y, Moulin P. Optimized feature extraction for learning-based image steganalysis. IEEE Transactions on Information Forensics and Security, 2007, 2(1):31-45.

[6] Westfeld A. F5 - a steganographic algorithm. The 4th International Workshop on Information Hiding. Binmingham: Springer, 2001:289-302.

[7] Sallee P. Model-based steganography. Digital Watermarking 2nd International Workshop, Seoul: Springer, 2004:154-167.

[8] Schaefer G, Stich M. UCID-An uncompressed colour image database. Technical Report, School of Computing and Mathematics, Nottingham Trent University, UK, 2003. 